\title{
Fast Segmentation of the Mitral Valve Leaflet in Echocardiography
}

\author{
Sébastien Martin ${ }^{1}$, Vincent Daanen ${ }^{1}$, Olivier Chavanon ${ }^{1,2}$, and Jocelyne \\ Troccaz $^{1}$ \\ 1 TIMC Lab, CAMI Team, \\ Institut d'Ingénierie d'Information de Santé (IN3S) \\ Faculté de Médecine - 38706 La Tronche cedex - France. \\ \{Sebastien.Martin, Vincent.Daanen, Jocelyne.Troccaz\}@imag.fr \\ http://www-timc.imag.fr/gmcao/index.html \\ Grenoble University Hospital, Cardiac Surgery Department, \\ 38043 Grenoble - France. \\ OChavanon@chu-grenoble.fr
}

\begin{abstract}
This paper presents a semi-automatic method for tracking the mitral valve leaflet in transesophageal echocardiography. The algorithm requires a manual initialization and then segments an image sequence. The use of two constrained active contours and curve fitting techniques results in a fast segmentation algorithm. The active contours successfully track the inner cardiac muscle and the mitral valve leaflet axis. Three sequences have been processed and the generated muscle outline and leaflet axis have been visually assessed by an expert. This work is a part of a more general project which aims at providing real-time detection of the mitral valve leaflet in transesophageal echocardiography images.
\end{abstract}

Keywords : Medical Image Analysis, Tracking and Motion, Active Contours, Ultrasound Imaging. 


\section{Introduction}

The mitral valve is one of the four valves of the heart; its function is to keep the blood flow in the physiological direction when the heart contracts. Due to various pathological factors, a mitral regurgitation can occur. The work presented in this paper belongs to a more general project of robot assisted surgery which aims at repairing a pathological mitral valve in a context of microinvasive beating heart surgery. The control of the robot is performed under ultrasound imaging guidance and required robust and real time algorithms to segment the valve. This project called GABIE is supported by the CNRS program ROBEA, and involves 4 laboratories (LIRMM, TIMC, LRP and CEA) and 2 University Hospitals (APH Paris and University Hospital of Grenoble).

Although transesophageal echocardiography is the classical imaging technique for mitral valve surgery, there is no satisfactory method allowing an automated segmentation of the valve.

The tracking of the myocardial border of the left ventricle (LV) is a very active research area that makes intensive use of deformable models, ([1],[2]), Markov random fields [3] or optical flow methods $([4],[5])$. Data processed are either in $2 \mathrm{D}+\mathrm{T}$ or $3 \mathrm{D}+\mathrm{T}([6],[7],[8],[9][10])$. [11] propose to use information fusion to track the LV in echocardiography in real-time. His algorithm requires a statistical shapes analysis of the LV, obtained by principal component analysis (PCA) on a large number of LV shape. We think, these methods will not work for the segmentation of the mitral valve leaflet, because of the high inter-patient variability. Mikic [12] uses active contours to segment either the left ventricle or the mitral valve leaflet. The method requires a manual segmentation on a image of the sequence at the beginning of the procedure and estimation of the optical flow field along the sequence. It takes about 20 minutes to process one complete cardiac cycle (i.e $\simeq 25$ to 30 images). [13] processes an image sequence using wavelet packet decomposition (in $2 \mathrm{D}+\mathrm{T}$ ) and then selects the sub-bands (in the wavelet domain) which preserve most of the energy of the target structure with an acceptable Signal to Noise Ratio. These sub-bands are then recombined to create the feature footprint ; this footprint is then used to analyze an image sequence. Although this method seems to process an image sequence fast, the analyzed sequences must not differ markedly from the data used to construct the filters.

In this paper, we present a semi-automatic method (a manual initialization is required) to segment the axis of mitral valve leaflet in transesophageal ultrasound images. The proposed approach uses 2 active contours. The method is designed to be fast and to achieve the segmentation in near real-time.

This work is intended to be the pre-operative step of the surgery scenario and should provide semi-automatic segmentation of several mitral cycles. In an intraoperative second step which is actually under development, the set of segmentation obtained during the pre-operative step will be used to detect the valve in real time. Therefore only near real time capability are required for the pre-operative algorithm, in order to make it usable in a surgical context and to achieve the 
repeatability condition of the mitral valve motion needed by the intra-operative step (more details are provide in conclusion).

\section{Material and Method}

\subsection{Context}

The mitral valve is a left-sided valve located between the left atrium and the left ventricle, made up of two fibrous membranes which are attached to the left ventricle muscle through the mitral annulus. On the free edges of the two leaflets, there are multiple strong cords (like parachute cords), in turn attached to papillary muscles (reinforcement of the left ventricle wall). When the heart contracts, the two leaves billow up to close off the opening between the left atrium and the left ventricle. The closure mechanism is mainly passive according to the pressure gradient between each side of the leaflet. During the contraction of the left ventricle there is also a geometrical modification of the shape of the annulus. Although the cardiac muscle motion resulting of the heart contraction is non rigid, it appear close to a rigid motion in one dimensional echocardiography images. Therefore two major kinds of movements can be shown in these images:

- the leaflet movement (main component) which is non rigid but relatively close to a rotation around a point based on the muscle-leaflet junction area called junction point,

- the muscle movement which is approximately rigid with essentially translational components and small rotational components;

These movements can be used as an a priori knowledge in order to facilitate the semi-automatic segmentation of the mitral valve in echocardiography.

\subsection{Method}

The proposed method relies on the use of two active contours (Figure 1) to track the leaflet efficiently : one tracks the cardiac muscle and the other tracks the mitral valve leaflet. The tracking method can be chronologically divided in

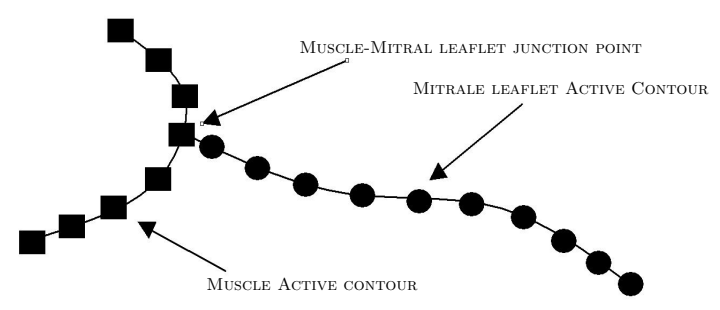

Fig. 1. The two contours 
2 times:

1. the segmentation of the cardiac muscle,

2. the segmentation of the mitral valve leaflet

Each segmentation is realized in two steps :

1. rough segmentation using a curve fitting algorithm.

2. refinement using active contours.

This method allow us to solve two reluctant problems of the mitral valve tracking:

- the ability to track very fast motions.

- the ability to separate the valve snake of the muscle during the opening valve phase.

The curve fitting algorithm providing rough segmentations, use measurements along curves normals. Therefore some 1D image processing techniques are required to detect feature points on curves normals.

This paper is organized as follows: section 3 describes the method used to build a rough segmentation of both mitral valve and cardiac muscle. Section 4 presents the proposed method to refine rough segmentation using snakes. Figure 2 presents the pipeline of our method, and sets up briefly the connection between different section of the paper.



Fig. 2. Synoptics of the proposed method 


\section{Rough Segmentation}

In this section, we explain how a rough segmentation of the cardiac muscle (resp. mitral valve leaflet) in the $k^{t h}$ image is computed using the final segmentation of the $(k-1)^{t h}$ image.

\subsection{Curve fitting algorithm}

The problem is to estimate the parameters of a transformation which minimizes the distance between two curves. Making the assumption that curves are defined by points stored in vectors: $Q=[x(s), y(s)]$, then the relation between two curves can be written as follows:

$$
Q_{f}=W X+Q_{i}
$$

where $Q_{f}$ is the target curve and $Q_{i}$ is the initial one. $W$ is the transformation matrix and $X$ the vector of the transformation parameters. The distance used is the sum of square normal measurements that can be approximated by:

$$
\begin{aligned}
\left\|Q_{f}-Q_{i}\right\|_{n}^{2} & =\frac{1}{N} \sum_{k=1}^{N}\left[\left(Q_{f}(k)-Q_{i}(k)\right) \cdot \boldsymbol{n}(k)\right]^{2} \\
& \approx \sum_{k=1}^{N} m(k)^{2}
\end{aligned}
$$

where $\|.\|_{n}$ denotes the norm based on normal measurements, $n(k)$ is the normal vector to the curve at the abscissa $k, m(k)$ is the normal measurement computed from $2 \mathrm{D}$ operator on normal profiles. Robustness to noise can be obtained by using a regularization term so that the problem can be expressed as:

$$
\hat{X}=\arg \min _{X}\left(\alpha\|X-\bar{X}\|^{2}+\left\|Q_{f}-Q_{i}\right\|_{n}^{2}\right)
$$

Solving Eq 4 in the least-squares sense is equivalent to a classical curve fitting problem. Blake has proposed a recursive algorithm to solve this problem in [14].

\subsection{Rough segmentation of the muscle}

As explained in section 2 the cardiac muscle motion appears close to a rigid motion in one dimensional images. Therefore, the rough contour position of the

cardiac muscle is estimated from the initial template (manual segmentation on the first image) by translating it.

The rough contour for the image $k$ is given by:

$$
\tilde{Q}_{k}^{m}=W^{m} X_{k}^{m}+Q_{0}^{m}
$$

where $X_{k}^{m}$ is the transformation estimated (i.e. a translation) and

$$
W_{0}^{m}=\left(\begin{array}{cc}
x_{0}^{m} & \mathbf{0} \\
\mathbf{0} & y_{0}^{m}
\end{array}\right)
$$


with $\mathbf{0}=(0,0, \ldots, 0,0)^{T}$

The curve fitting algorithm requires feature detections on the curve normals. Therefore it is necessary to process the gray-level profiles corresponding to these directions in order to get the normal measurements.

A canny edge detector approximated by the derivative of the Gaussian kernel $(\sigma=1.4)$ is used for this purpose.

\subsection{Mitral Valve Leaflet Transformation Estimation}

In this section, the abscissa of the junction point on the muscle curve at time $k-1$ is supposed to be given. It will be used as a rough estimate of the current abscissa of the junction point. Given that the leaflet motion is close to be a rotation around the junction point, this point must be invariant to the involved transformation. For this purpose, the previous segmentation of the leaflet (after refinement) is first translated on the junction point and then an affine transformation without translations components is used for fitting. All computation are made in a coordinate system centered on the junction point. In this way the junction point is a fixed point (invariant to the involved transformation). The computation of $\tilde{Q}_{k}^{v}$ is then given by:

$$
\tilde{Q}_{k}^{v}=W_{k-1}^{v} \cdot X_{k}^{v}+\underline{Q_{k-1}^{v}}
$$

where:

$$
W_{k-1}^{v}=\left(\begin{array}{cccc}
x_{k-1}^{v} & \mathbf{0} & \mathbf{0} & y_{k-1}^{v} \\
\mathbf{0} & y_{k-1}^{v} & x_{k-1}^{v} & \mathbf{0}
\end{array}\right)
$$

and represents a $2 \mathrm{D}$ affine transformation matrix ; and $Q_{k-1}^{v}$ represents the mitral valve leaflet contour of the $k-1^{t h}$ image translated to the rough junction point of the $k^{\text {th }}$ image.

The presence of the mitral valve leaflet is characterized by a ridge in the image, that corresponds to a local maximum in the gray-level profiles along the normal directions. The second order derivative of a 1D Gaussian kernel is used to detect the valve on normal curves. In fact this operator is a good measurement of the contrast between two regions.

\section{Refinements}

\subsection{Need for a refinement}

At the end of the previous step, the contours are in a neighborhood of feature structures. Thus we have to bring them closer to the cardiac muscle (resp mitral valve leaflet) ; This is done by using active contours.

Snakes has been originally introduced by Kass and al [15]. A snake contour is described parametrically by $v(s)=(x(s), y(s))$ where $x(s), y(s)$ are $x, y$ coordinates along the contour (the so-called snaxels) and $s \in[0,1]$ is the normalized 
parametrization. The snake model defines the energy of a contour $v(s)$, as:

$$
E_{\text {snake }}=\int_{s=0}^{1} \lambda E_{\text {Int }}(s)+E_{E x t}(s) d s
$$

where $E_{\text {Int }}$ is the internal energy of the contour, imposing continuity and curvature constraints, and where $E_{E x t}$ is the image energy allowing the snake to move to the feature points in the image. $\lambda$ is the regularization parameter governing the compromise between adherence to the internal forces and adherence to the external forces. An initial contour evolves by minimizing of the Equation 1.

\subsection{Muscle Snake Energy Definition}

The energy of the muscle snake is composed of two terms related to the internal energy and one term related to the external energy.

Internal Energies The $1^{\text {st }}$ term is related to the length of the curve. It penalizes curves where the distance between two successive snaxels is far to a distance $d_{m}$ which is computed at the beginning of the algorithm. This energy keeps the length curve close to the initial one, and prevents the snake to segment the whole cardiac muscle. This $1^{\text {st }}$ term is written:

$$
E_{\text {Int }_{1}}^{m}\left(s_{i}\right)=\alpha_{1}^{m}\left(\left|s_{i-1}-s i\right|-d_{m}\right)^{2}
$$

where $\alpha_{1}^{m}$ is a weighting parameter.

The $2^{\text {nd }}$ internal energy term approximates the $2^{\text {nd }}$ order derivative of the curve. This term is computed from the 'cardiac muscle' control points $s_{i}^{m}$ by using the finite differences:

$$
E_{\text {Int }_{2}}^{m}\left(s_{i}\right)=\alpha_{2}^{m}|s(i-1)-2 s(i)+s(i+1)|
$$

where $\alpha_{2}^{m}$ is a weighting parameter.

External Energy The external energy term is related to the modulus of the gradient image. It allows the contour to move toward the cardiac muscle appearing as an edge in the image. This energy term is given below:

$$
E_{E x t}^{m}\left(s_{i}\right)=-\left\|\Delta I\left(s_{i}\right)\right\|^{2}
$$

\subsection{Mitral Snake Energy Definition}

Because the movement of the mitral valve leaflet is a bit more complex than the movement of the cardiac muscle, we introduce different energy terms.

Internal Energies The internal energy is composed of the same energy terms that for the muscle snake with weighting parameters $\alpha_{1}^{v}$ and $\alpha_{2}^{v}$ respectively for the length constraint and the curvature constraint. 
External Energy The image of the leaflet corresponds to a local maximum of intensity. Reasoning about the image intensity map as a surface in $\mathbf{R}^{3}$, features corresponds to areas where one of the principal curvatures is high (Figure 3). The image of the higher principal curvature allows us to build a robust external energy function:

$$
E_{E x t}^{v}\left(s_{i}\right)=K^{+}\left(s_{i}\right)
$$

where $K+$ is the higher principal curvature.

In the actual implementation, principal curvatures are computed from eigenvalues of the Hessian matrix of the image intensity map.

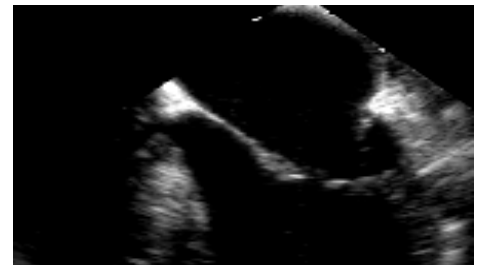

a)

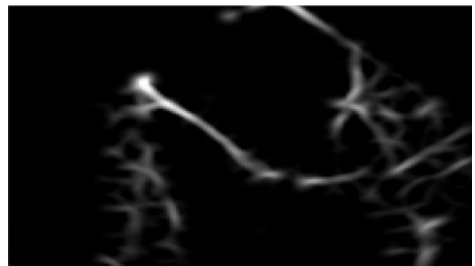

b)

Fig. 3. a) Ultrasound image b) $K^{+}$of the image

\subsection{Dynamic Programming Minimization (DP)}

The problem of energy snake minimization is solved using dynamic programming (DP). Amini has proposed this method [16] in order to overcome the limitations of classical variational methods like instability or non optimality and to allow the use of stronger constraints without falling into a local minimum. When an energy functional can be written as:

$$
E_{\text {snake }}=\sum_{i=1}^{N-2} E_{i}\left(s_{i-1}, s_{i}, s_{i+1}\right)
$$

the minimization can be obtained using Dynamic programming as described in [16].

The algorithm is iteratively applied to the contour. At each iteration, a snaxel can move in a previously defined neighborhood (search area). DP allows us to correctly minimize the energy function and provides a way to locate the junction point on the muscle curve. The search-area for each snaxel of the muscle snake is defined by a 8-neighborhood around the snaxel. We define the same search-area for all snaxels of the mitral snake, except for the one (junction point) which is connected to the muscle snake. For this one, the search area is defined by a region of 8 pixels around its previous estimate and on the muscle snake curve. We do 
not define an external energy term for this point. In this way, the junction point will be only driven by its internal energy according to the position of the two next snaxels and will be correctly adjusted to the muscle curve by continuity of the leaflet curve.

\section{Results and Discussion}

\subsection{Algorithm Initialization}

The algorithm is initialized by the manual segmentation of both the muscle and the mitral valve leaflet and by the specification of the junction point. The algorithm then computes $d_{m}\left(\operatorname{resp} d_{v}\right)$ the mean distance between two successive points of the muscle curve (resp of mitral curve). Manual segmentations influences the robustness and the accurency of segmentations. We have observed that segmenting a large part of the cardiac muscle during the initialization improve the robustness of the algorithm.

\subsection{Results}

The described method has been implemented using Matlab ${ }^{\circledR}$ (and in Matlab language). Dynamic programming algorithms has been coded in $\mathrm{C}$ using mex. Snakes requires the tuning of some parameters : $\left(\alpha_{1}^{m}, \alpha_{2}^{m}\right)$ for the muscle snake and $\left(\alpha_{1}^{v}, \alpha_{2}^{v}\right)$ for the mitral snake.

The processing time of our algorithm depends on the number of snaxels used. In a general rule to obtain accurate segmentations, the mean processing time is less $0.5 \mathrm{~s}$ and the number of iteration for the DP algorithm less than 10 .

The validation of the method is based on the visual assessment of an expert. We have processed 4 sequences of 250 to 300 images. For each one, the medical expert is asked to segment the cardiac muscle, the mitral valve leaflet and to point the junction point. The sequence is then processed with the proposed method. The medical expert is then asked to give his opinion about the segmentation proposed by the method. Most of the time (about $90 \%$ of the images), the expert is very confident in the segmentation provided by the method. In the remaining $10 \%$, although the segmentation is "bad" (and not absolutely false !), the snake reconverges to a good contour in the next 3-to-6 images.

\subsection{Discussion}

The proposed method still has some drawbacks. The main one is the tunning of parameters. However the main objective of our algorithm is to provide a set of segmentations that will be used during the intra-operative step where no tunning of parameters is necessary. So, we could imagine trying several parameter configurations in order to obtain a good tunning. Another point which could be improved is the way we compute the principal curvatures which is known to be noise sensitive [17] ; We are very confident in the near real-time possibilities of 

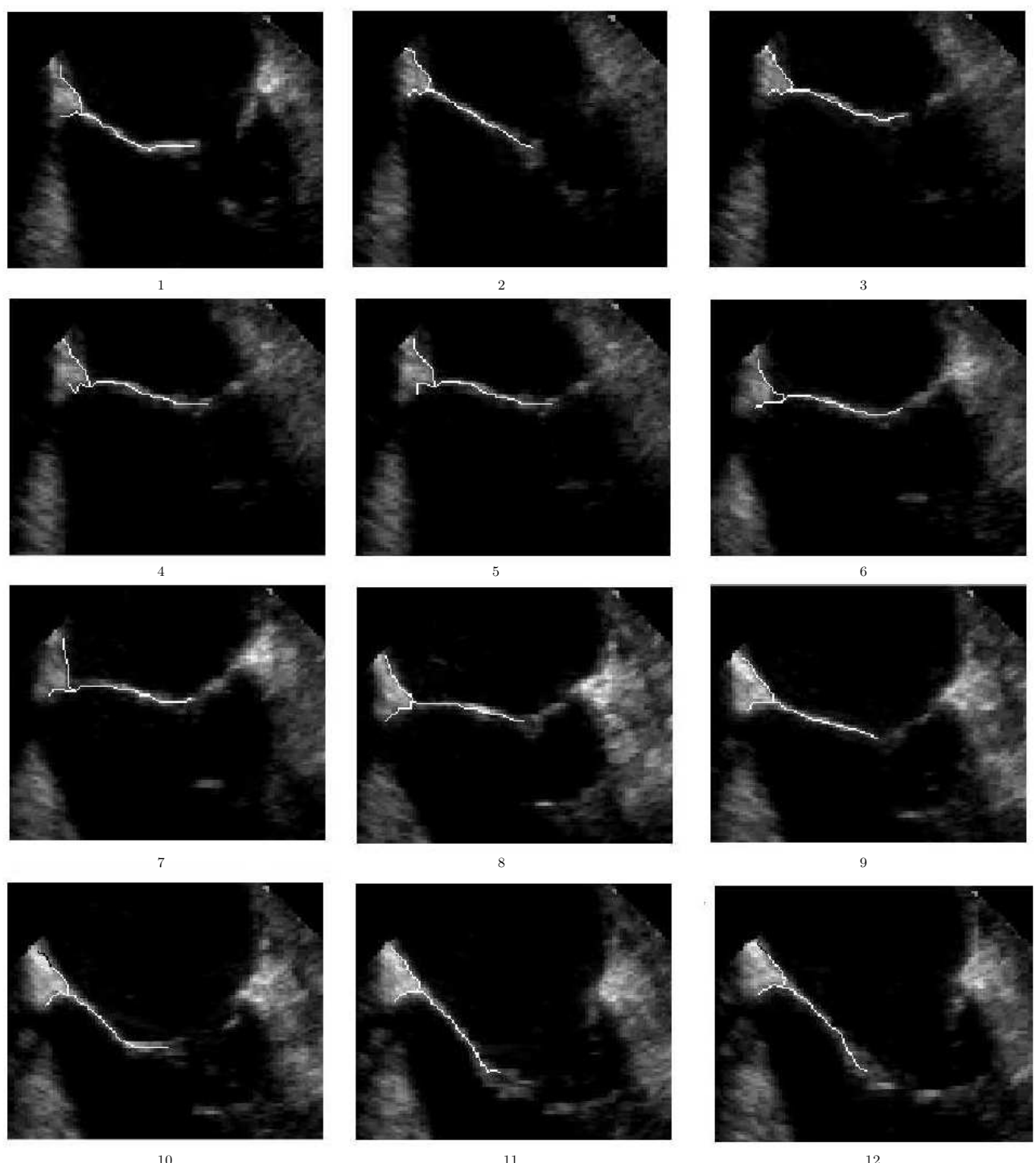

Fig. 4. Processing of a sequence. Segmentation of the biggest leaflet 
this algorithm needed for the surgical scenario (sec. 1). From our experience, converting algorithms from Matlab language to ' $\mathrm{C}$ ' language should divide the processing time by a factor of 10 . Other 'coding' optimizations such the dynamical selection of a ROI to process (instead of processing the whole image) should speed up the image processing and thus allow a near real-time mitral valve leaflet detection.

\section{Conclusion and future work}

In this paper, a method to rapidly segment the mitral valve leaflet in echocardiography has been presented. Due to the fact that the motion of the valve and the motion of the muscle are quite different, we use two contours to capture these motions. For each contours segmentations are realized in two step: first we use a curve fitting technique to provide rough segmentation of the tracked contour then we refine it by using a snake. As mentioned in the abstract, this work is the first step of a two-steps procedures which aims at segmenting the mitral valve leaflet in echocardiagraphic images. The presented method will be the preoperative step of the surgery scenario and will provide segmentation of several cardiac cycles. During the intra-operative step, we should be able to segment in real time the cardiac muscle and the mitral leaflet by searching the more similar image in the pre-operative images and then applying a refinement to accurately segment the mitral valve leaflet.

\section{References}

1. Cootes, T., Taylor, C.: Active shape models - smart snakes. Proceedings of the British Machine Vision Conference" (1992) 266-275

2. Chalana, V., Linker, D.T., Haynor, D.R., Kim, Y.: A multiple active contour model for cardiac boundary detection on echocardiographic sequences. IEEE Trans. Med. Imag 15 (1996) 290-298

3. Mignotte, M., Meunier, J., Tardif, J.C.: Endocardial boundary estimation and tracking in echocardiographic images using deformable templates and markov random fields. Pattern Analysis and Applications 4 (2001) 256-271

4. Mailloux, G.E., Langlois, F., Simard, P.Y., Bertrand, M.: Restoration of the velocity field of the heart from two-dimensional echocardiograms. IEEE Trans. Med. Imag 8 (1989) 143-153

5. Adam, D., Hareuveni, O., Sideman, S.: Semiautomated border tracking of cine echocardiographic ventricular images field of the heart from two-dimensional echocardiograms. IEEE Trans. Med. Imag 6 (1987) 266-271

6. Jacob, G., Noble, J., Behrenbruch, C., Kelion, A., Banning, A.: A shape-spacebased approach to tracking myocardial borders and quantifying regional leftventricular function applied in echocardiography. IEEE Trans. Med. Imag 21 (2002) 226-238

7. andY. Chen, A.A., Elayyadi, M., Radeva, P.: Tag surface reconstruction and tracking of myocardial beads from spamm-mri with parametric b-spline surfaces. IEEE Trans. Med. Imag 20 (2001) 94-103 
8. Lorenzo-Valds, M., Sanchez-Ortiz, G.I., Mohiaddin, R.H., Rueckert, D.: Atlasbased segmentation of $4 \mathrm{~d}$ cardiac mr sequences using nonrigid registration. (2002)

9. Mitchell, S., Lelieveldt, B.P., van der Geest, R., Bosch, H.G., Reiber, J.H., Sonka, M.: Time-Continuous Segmentation of Cardiac MR Image Sequences Using Active Appearance Motion Models. Volume 4322 of SPIE Medical Imaging., Tokyo, Japan (2001) 249-256

10. Montillo, ., Metaxas, D., Axel, L.: Automated Segmentation of the Left and Right Ventricles in 4D Cardiac Spamm Images. Medical, Image Computing and Computer Assisted Intervention (MICCAI), Tokyo, Japan (2002) 620-633

11. Comaniciu, D., Zhou, X., andY. Chen, S.A., Elayyadi, M., Radeva, P.: Robust Real-Time Myocardial Border Tracking for Echocardiography : An Information Fusion Approach. IEEE Trans. Med. Imag 20 (2001) 94-103

12. Mikic, I., Krucinski, S., Thomas, J.D.: Segmentation and tracking in echocardiographic sequences: active contours guided by optical flow estimates. IEEE Trans. Med. Imag. 17 (1998) $274-284$

13. Jansen, C., Arigovindan, M., Sühling, M., Marsch, S., Unser, M., P., H.: Multidimensional, multistage wavelet footprints: A new tool for image segmentation and feature extraction in medical ultrasound. In Sonka, M., Fitzpatrick, J., eds.: Progress in Biomedical Optics and Imaging, vol. 4, no. 23. Volume 5032 of Proceedings of the SPIE International Symposium on Medical Imaging: Image Processing (MI'03)., San Diego CA, USA (2003) 762-767 Part II.

14. A, B., M, I.: Active Contour. Springer-Verlag, Berlin Heidelberg New York (1998)

15. Kass, M., Witkin, A., Terzopoulos, D.: Snakes : Active contour models. International Journal of Computer Vision 1 (1988) 321331

16. Amini, A.A., Weymouth, T.E., Jain, R.C.: Using dynamic programming for solving variational problems in vision. IEEE Trans. Pattern Anal. Mach. Intell. 12 (1990) $855-867$

17. Lachaud, J.O., Taton, B.: Deformable model with a complexity independent from image resolution. Computer Vision and Image Understanding (2005) Accepted. To appear. 